\title{
Sobre la gobernanza digital, política digital y educación*
}

\section{On digital governance, digital policy and education}

\author{
Luis Alex Valenzuela-Fernández** \\ Zoila Mercedes Collantes-Inga*** \\ EdUARDo ERnesto DURAND-Hipólito****
}

\section{Resumen}

Objetivo. El presente artículo tiene por objetivo realizar una revisión acerca de los fundamentos referidos a aspectos claves de la política digital y sus mecanismos de gobernanza. Metodología. Se ha optado por emplear la metodología hermenéutica a fin de abordar cualitativamente la temática con base en el análisis bibliográfico. Resultados. Se describen los mecanismos y procesos inherentes a la gobernanza digital a niveles micro y macro y su influencia en el orbe, así como el desarrollo de las políticas digitales aplicadas al campo de la educación. Conclusión. Se discuten las oportunidades y dificultades del desarrollo de los sistemas de gobernanza multinivel con base en la aplicación de infraestructuras digitales y la consolidación de ecosistemas tecnológicos urbanos.

Palabras clave: gobernanza digital, ciudadanía digital, gobernanza de datos, política digital, neoliberalismo digital, ciudad inteligente.

\begin{abstract}
Objective:The objective of this article is to conduct a review of the fundamentals related to key aspects of digital policy and its governance mechanisms. Methodology: The hermeneutic methodology has been chosen in order to qualitatively approach the topic, based on bibliographic analysis. Results: The mechanisms and processes inherent to digital governance at micro and macro levels and their influence in the world are described as well as the development of digital policies applied to the field of education. Conclusion: The opportunities and difficulties of developing multilevel governance systems based on the application of digital infrastructures and the consolidation of urban technological ecosystems are discussed.
\end{abstract}

Key words: digital governance, digital citizenship, data governance, digital policy, digital neoliberalism, smart city.

\footnotetext{
*Artículo de revisión, parte del proyecto de investigación "Implicancia de las políticas digitales y las tecnologías en la sociedad contemporánea”.

** Universidad Nacional Mayor de San Marcos. Lima, Perú. E-mail: lvalenzelaf@unmsm.edu.pe

(D) orcid.org/0000-0002-8743-4092 Google Scholar

**** Universidad Privada del Norte. Lima, Perú. E-Mail: zoila.collantes@upn.pe

(D) orcid.org/0000-0002-8741-9263 Google Scholar

***** Universidad Nacional Mayor de San Marcos. Lima, Perú. Email: edurand.610@gmail.com

(D) orcid.org/0000-0003-1194-9724 Google Scholar
} 


\section{Introducción}

Uno de los dilemas actuales, producto de la rápida expansión de las tecnologías de grandes datos en el quehacer cotidiano, está referido a comprender la compleja relación entre la recopilación y el procesamiento de grandes volúmenes de datos personales, la aparición de novedosas formas de gobernanza y vigilancia digital que genera nuevos retos de entendimiento y difusión en la ciudadanía. Cada vez hay un número creciente de docentes, administradores y de organismos de financiamiento que están reconociendo la trascendencia de las aplicaciones digitales en humanidades en relación con la misión docente, sea en colegios, institutos o universidades, y su rol clave para la promoción de la equidad y los principios democráticos de la sociedad en general (Gibson, 2019). El papel central que desempeña la ciudadanía, desde la óptica de los estudios sociales, hace imprescindible comprender cómo definir y reinterpretar el concepto de ciudadanía en la era de Internet (Choi, 2016). La importancia de comprender el cómo definir la ciudadanía digital y cómo la misma está cambiando a la par del desarrollo e implementación más álgida de las tecnologías digitales en las actividades cotidianas, permitirá una mejor educación de la ciudadanía en esta floreciente era de internet ( ${ }^{\mathrm{a} S}$ chou \& Hjelholt, 2018; Choi, 2016; Williamson, 2015, 2016).

La disminución de las brechas digitales en la población es un proceso creciente en relación al caso latinoamericano, tal como lo revela el estudio realizado por Ovando, Olivera y Bocarando (2018) en relación al progreso digital en la sociedad mexicana, donde se concluyó que luego de aplicarse una reforma de más de una década para subsanar la enorme brecha digital en su contexto, resultó no ser totalmente homogénea, pues según dichos investigadores se deberán requerir mayores y decisivas acciones para atender la inclusión digital de las mayorías, en especial de las poblaciones de menores recursos que siempre son postergados en los procesos de innovación (Evans \& Gomes, 2017).

Hansen (2019) explicó que la revolución digital ha gestado un enorme impacto en la génesis y transformación del sector empresarial privado, donde las plataformas digitales y la disminución de las barreras de acceso dan una nueva vida a los crecientes entornos corporativos en la actualidad.

\section{Discusión}

\section{Gobernanza desde el contexto digital}

El campo de las nuevas tecnologías del manejo de datos aparece sobre el escenario mundial como ejes promisorios de una nueva era de precisión y objetividad en la política y la gobernanza, científicamente informadas para la sociedad de corte digital (Williamson \& Piattoeva, 2018). 
Como es normal y cotidiano en los sistemas de administración occidentalizados, estos giran en torno al sistema privado o privatizado, donde los principales activos urbanos tales como la energía y las comunicaciones se encuentran regidas por consorcios privados, y algo similar es lo relacionado con la accesibilidad a los datos y sus limitaciones, tanto a escala como en volumen (Zhao, 2017); pero que en relación a los procesos de gestión documentaria en las agencias públicas se puede acceder bajo un enfoque determinado por parámetros de una gestión urbana integrada y coordinada en tiempo y espacio, tal como lo expusieron Ovando et al. (2018). También cabe resaltar sobre este punto que en el contexto de la gobernanza digital se han implementado una serie de trasuntos relacionados al plano neoliberal (Nosthoff \& Maschewski, 2019; Zalnieriute \& Milan, 2019; Pérez \& Foronda, 2018; 'Schou \& Hjelholt, 2018; Gil-García, Dawes \& Pardo, 2017; Sixin, Yayuan, Jiang \& Yue, 2017; Marland, Lewis \& Flanagan, 2016). La neoliberalización de las políticas digitales se sustenta en el rol proactivo del Estado en la transformación de su política y políticas, principalmente a través del empleo de instrumentos de medición e indicadores (Giannone \& Santaniello, 2018).

Respecto al mecanismo de gobernanza empleado en el contexto actual, sobre qué doctrina es la que se está aplicando, Aili y Nilsson (2016) mencionaron en su trabajo que los profesionales del área expuestos a una creciente gobernanza y sus mecanismos derivados tales como documentación, legislación, enfoque de desempeño, entre otros, se sustentan en mecanismos de gobernanza neoliberal. Sin embargo, según lo argumentado desde el contexto danés por ${ }^{\text {b}} S$ chou \& Hjelholt (2018), sobre las investigaciones existentes acerca de la ciudadanía digital, aún no logran establecer conexiones de manera sostenida, ya que todavía no hay un panorama claro sobre las políticas que deben agenciarse al ciudadano digital; a pesar de la concepción de ciudadano digital neoliberalizado que se encuentra sujeto a poder ejercer sus potencialidades digitales.

La Internet debe entenderse en su aplicabilidad en el campo social, y ello dependiendo de su empleo, como herramienta o mecanismo que puede desestabilizar o desafiar al gobierno o en el mejor de los casos el instrumento que acuda en su auxilio al ayudarlo a interconectarlo, especialmente a las autoridades locales, con los ciudadanos, o al menos servir como una herramienta para que el Estado involucre a la ciudadanía (Han \& Jia, 2018). Al respecto, pensar en la forma y el significado de las transformaciones digitales, Flyverbom, Deibert \& Matten, (2019) mencionaron que deben considerarse dimensiones tan diferentes como el tamaño y el dominio de los gigantes de Internet, la disrupción de los mercados y las industrias, la naturaleza de las nuevas adquisiciones y los monopolios. Este último aspecto es de crucial importancia para poder gestionar plataformas que se fusionen a sistemas digitales masivos requeridos para una adecuada administración del flujo de datos administrativos, de evaluación y del personal, que son de gran utilidad en la gestión y control de sistemas, instituciones e individuos (Williamson, 2016). Al respecto, Constantinides, Henfridsson y Parker (2018) mencionaron que la plataforma digital en sí misma no necesariamente tiene activos físicos en forma de recursos de infraestructura, ni generar valor a través de la venta de producto; motivo 
por el cual su implementación conlleva a un nivel de desempeño orientado a la optimización, tal como lo manifestaron da Silva, Ribeiro, Soares y do Nascimento (2019) en un trabajo realizado en Brasil acerca de la aplicación de las nuevas tecnologías al campo de la gobernanza, cuyos resultados fueron muy promisorios ya que promovieron el alineamiento continuo de los procesos de las organizaciones en el marco digital como hecho trascendente en el proceso de gestión organizacional. Resultados similares se exhibieron con antelación por Botchway, Boateng y Kwofie (2016) en Ghana, donde apuestan por el gobierno electrónico, a pesar de que implica múltiples etapas de desarrollo, puede generar muchos beneficios para los diversos sectores del país.

Respecto a un punto de vista diferente, Clarke, Lindquist y Roy (2018) manifestaron: ¿Cómo están afectando las tecnologías digitales a la gobernanza democrática en Canadá, y cómo podría la era digital reforzar o socavar nuestras instituciones públicas y nuestra capacidad colectiva de resolución de problemas en los próximos años? Las empresas privadas deben someterse a la legislación de los países en los que ofrecen servicios, donde dichas leyes pueden variar ampliamente e influir en diversos aspectos de los servicios, desde la eliminación de contenido hasta el manejo, la retención y el intercambio de datos de los usuarios, hasta la censura y la vigilancia (Flyverbom et al., 2019).

Por medio del flujo continuo de datos abiertos las condiciones de buen gobierno, bajo dichos parámetros, deberá encontrarse vinculado con procesos exitosos de emprendimiento digital, la vitalidad de un sector tecnológico local y la integración exitosa de los activos de datos públicos en servicios de software patentados (Barassi, 2019; Barns, Cosgrave, Acuto \& Mcneill, 2016). De acuerdo con la tendencia del big data, ello se ha establecido ampliamente en China donde se formó un sistema comercial heterogéneo por medio del cual se generaron datos masivos en diversos formatos, por lo que se requirió el surgimiento de un nuevo patrón de gobernanza basado en el tratamiento de los recursos de información del gobierno, es decir, los centros de servicios de big data y el manejo de archivos inteligentes que derivarán en bucles para almacenamiento, acceso, utilización y preservación a largo plazo de recursos de data gubernamental (Ju, Liu \& Feng, 2019; Singh, 2019; Barreiro, 2018; Sixin et al., 2017).

El modelo de ciudad interactiva, en tiempo real, donde el empleo de datos en tiempo real pone de manifiesto los detalles sumamente complejos de sistemas de redes a pequeña y gran escala sobre las que se sustentan actividades tales como transporte, comercio electrónico, entre otras; son muy obvias que están al servicio de la comunidad de dicho entorno en su conjunto (Silva, Khan \& Han, 2018; Flyverbom et al., 2019; Barns et al., 2016; Botchway et al., 2016) y que bajo dicho parámetro tiende a obcecar con una óptica sencilla y simplista sobre la relación causal que ocurre entre el acceso a la data y las tomas de decisiones a nivel urbano interactivo; mas dicho servicio en relación al ciudadano no debe ser percibido como algo rutinario o simple, sino que el acceso al conjunto de datos integrados en tiempo real debe asumirse desde 
Sobre la gobernanza digital, política digital y educación

la acción ejecutiva del ente encargado, con un sesgo hacia la población, concibiéndose este aspecto como un límite administrativo de acceso a información sobre aquellos datos a los que no se les ha asignado los respectivos niveles de accesibilidad (Barns, 2016). Sobre este punto, hay que tomar el ejemplo del modelo chino que, bajo un marco de políticas trazadas para un gobierno electrónico, ha delimitado diversos niveles y rubros a fin de gestionar una mejor y más eficiente capacidad administrativa, con la respectiva prestación de servicios a la ciudadanía y su involucramiento al sistema (Han \& Jia, 2018).

La política de la ciudad digital se estructura en torno a un panorama de gobernanza altamente diferenciado. Las ciudades inteligentes integran varios subsistemas en la capa de aplicación para ofrecer servicios oportunos y confiables (Silva et al., 2018). Lo referente a los datos públicos, y cómo se utilizan, ha sido un área muy fructífera de investigación que, a pesar de existir en el medio varios trabajos al respecto, continúa en un proceso de evolución constante (Felix, Rauschnabel \& Hinsch, 2017; Barns, 2016; Choi, 2016). Ahora bien, si se asumen de forma conveniente el aspecto político-económico, se debe de dar una aproximación a los mecanismos que permiten la implementación y costes del almacenamiento de datos, según sea los requerimientos del sistema y la aplicabilidad de los mismos; ya que si bien existen programas de data abierta al público, estos deberán encontrarse en un formato conveniente y amigable con los usuarios y legibles por los sistemas empleados (Spante, Hashemi, Lundin \& Algers, 2018), con la finalidad de implementar el desarrollo promisorio de más mercados sustentados en activos de datos públicos, que algunos investigadores como Paschoal y Wegrich (2019), bSchou y Hjelholt (2018), Williamson \& Piattoeva (2018) o Zhu y Peters (2018) han tenido a bien en denominar como mecanismos de gobernanza digital por código. Quizás más que promover la responsabilidad del gobierno y el compromiso orientado a los ciudadanos, está la reconstitución del papel del gobierno pueda ser el mayor legado de los programas de datos abiertos (Barns, 2016).

Otro aspecto en tomar en cuenta en relación a la gobernanza digital es el asumir la idea del ciudadano digital y la ciudadanía digital. Al respecto, Han \& Jia (2018) explicaron que la Internet puede haber transformado la interacción entre el Estado y la sociedad en China, especialmente al empoderar a los ciudadanos; lo que resulta altamente significativo en visos de concretar una ciudadanía digital, ya que los avances progresivos y acelerados de las tecnologías digitales contribuye a cambios estructurales masivos que repercuten en la economía global (Hansen, 2019). Por su parte Akcil, Altinay y Altinay (2016) se refirieron sobre la idea tradicional de ciudadanía limitada por las fronteras, superpuesta por el concepto de la ciudadanía digital, en la cual todos los ciudadanos del mundo puedan emplear similares plataformas digitales, ya que al encontrarse en capacidad de emplear las novedosas tecnologías digitales, en diversas locaciones en el planeta, pone en evidencia que todos los ciudadanos del globo pueden ser considerados ciudadanos digitales y con ello gozar de derechos y responsabilidades iguales (Bellanova, 2016). Las ideas normativas específicas vinculadas a la ciudadanía digital mediante 
la formulación de políticas nacionales muestran cómo se ha manifestado en el marco legal y tecnológico, derivando a nuevas prácticas gubernamentales puedan implementarse en las instituciones existentes del sector público, ya que la reestructuración del modo de gobernanza permite la consolidación de la ciudadanía digital (Knox, Williamson \& Bayne, 2019; Huygh \& De Haes, 2019; Pérez \& Foronda, 2018; Felix et al., 2017).

Con relación a la temática sobre la exclusión social, que según lo expuesto por Fuente (2017), se toma como desafío en todas las agendas de política social, sean de carácter nacional o internacional (Echalar e Peixoto, 2017). Además, en relación con la brecha digital, puede mencionarse una serie de situaciones variopintas, tales como la de los individuos con discapacidad (Fuente, 2017; Hepburn, 2018), en especial los niños, han tenido y tienen dificultades para materializar sus derechos en la era digital, al igual que sus sociedades, ya que son pasibles de vulnerabilidad por lo que requieren de apoyo para un acceso con equidad a las tecnologías digitales (Alper \& Goggin, 2017). Igualmente, frente a la situación de los migrantes digitales, el cerrar la brecha digital en torno de los mismos y su adaptación al panorama cambiante de la ciudadanía digital (Salemink, Strijker \& Bosworth, 2017), es otro dilema de gran envergadura al cual los europeos a través de una serie de medidas (programa europeo Horizonte 2020), integran una enorme masa de individuos a fin de empoderarlos en relación con las nuevas tecnologías (Janowski, Estevez \& Baguma, 2018; Neufeld \& Delcore, 2018; Fuente, 2017).

\section{Gobernanza de la educación bajo la política digital}

Un hecho trascendente acerca de la investigación educativa se encuentra, en gran medida, desconectada del análisis de los estudios sociológicos de datos, especialmente aquellos que se sustentan en la sociología de la cultura y práctica digitales que, según lo estipulado por Ozga (2015), buscan la apertura de "la caja negra" de los datos digitales para develar el quid del trabajo político que facilitan el tratamiento de datos, a fin de definir lo conocido y conocible en la sociedad contemporánea. Williamson (2015) puso de relieve que lo relacionado con las tecnologías de bases de datos digitales son herramientas imprescindibles para generar, analizar y difundir una cantidad de data necesaria para gobernar la educación (Bianchi \& Sousa, 2016). Por ejemplo, en relación con la información estadística, que guarda cierta apariencia de objetividad, son elementos que se integran positivamente en relación con el establecimiento y formulación de políticas educativas, asumiendo los activos escolares como aulas y escuelas, como plataformas de datos para reconfigurar la realidad educativa en data numérica que aporte al análisis de datos para poder seguir ajustando mecanismos y políticas cada vez más efectivas (Emejulu \& McGregor, 2016).

Ozga (2015), realizó un intenso análisis crítico de diversos trabajos cuestionando a su vez los estándares de muchos de ellos en relación al tratamiento de datos digitales en relación 
a los procesos educativos o escolaridad, estimando parámetros tales como la ponderación, compilación, análisis e informes generados en dichos contextos que estuvieron orientados a la comprensión y optimización de los procesos de aprendizaje y los entornos donde este proceso se desarrolla, empleando para ello técnicas modernas tales como la minería de datos y tratamiento de grandes volúmenes de data que en los supuestos se encuentran supeditados a mejoras sustantivas a nivel de eficiencia, competitividad al momento de evaluar el desempeño de alumnos y maestros.

Según la percepción del manejo de grandes volúmenes de información digital que va desde la generación, aglomeración, procesamiento y tratamiento analítico que se hayan generado a nivel local, regional, nacional o internacional permitirán soluciones viables a los problemas del campo de la escolarización (Maltese \& Giunchiglia, 2016). Lo que en un principio se estimó como una labor de conocimiento de la realidad de un entorno y su problemática, abordada desde las medidas políticas más convenientes, en la actualidad se subordina a patrones de datos e información generados y del cómo se materialice su interpretación, hecho que se establece desde el sustento técnico de soportes informáticos y los mecanismos de codificación de la data generada para utilizarse según se estime conveniente. De este aspecto, nace cómo situar la discusión de los datos dentro de un marco conceptual más amplio de la problemática de gobernar y su relación con el conocimiento. "Educational digital data are not novel in themselves" [Los datos digitales educativos no son novedosos en sí mismos] (Williamson, 2015, p. 16). Según dicho autor, todo lo referente a la recopilación y digitalización del conjunto de datos educativos de carácter masivo tiene una vasta historia que abarca ya casi un siglo. Sobre dicho proceso, se recalca que ello no es tan significativo como el tratamiento de datos digitales que va desde el análisis, visualización, predicción y prescripción para ejercer un gobierno educativo digital contemporáneo que sea maleable en función del empleo de herramientas funcionales de gestión de políticas públicas emergentes que pueda aprovechar de forma significativa elementos como las redes sociales y la masividad de datos. La gobernanza educativa precisa con mayor avidez de la exploración de soportes de datos socio-técnicos e instrumentos de política digital de carácter operativo.

De acuerdo con Williamson (2015), los asuntos relacionados con la educación, a pesar de que generan una gran cantidad de data, son cada vez más fáciles de ser rastreados, minados y esquilmados sin el más mínimo esfuerzo. Siempre es posible rastrear datos legibles por medio de algún sistema desarrollado por alguna compañía. A pesar de que estas prácticas no son novedosas en el contexto europeo, permite enmarcar un derrotero adecuado de una forma novedosa de praxis de gobierno educativo, distinto a lo conocido, ya que se afianza cada vez más el empleo de políticas sustentadas en datos digitales a fin de conseguir una adecuada auditoría de forma constante, orientada a monitorear las acciones estudiantiles a tiempo real y tomar medidas susceptibles de intervención pedagógica. Por consecuencia, el examen de los asuntos relacionados con la gobernanza educativa se sustenta en la exploración de soportes e 
infraestructuras de datos y la generación de instrumentos de políticas digitales que lo harán cada vez más efectivo en sus operaciones (Flyverbom et al., 2019).

Según lo manifestado por Decuypere (2016), la gobernanza educativa es cada vez más afecta a los mecanismos sustentados en medios digitales, ya que el campo de las oportunidades y sus posibilidades en función de los dispositivos digitales se han puesto al servicio de las organizaciones políticas tanto local como internacionalmente, tal como es el caso del panorama europeo que ha tenido a bien en la revaloración del rol funcional de los dispositivos digitales (Dahya, 2016); de forma especial, aquellos basados en plataformas web en relación a la gobernanza educativa de dicha región donde se han adaptado una vastedad de dispositivos digitales como servidores web, bases de datos digitales de acceso abierto, sitios web, entre otros, como herramientas eficaces para la distribución del conocimiento educativo a los interesados (Cushing \& Shankar, 2018) y con ello la participación efectiva de expertos que permiten la difusión y acercamiento a dichas políticas que se han adoptado de acuerdo con las necesidades del público. Al respecto, su análisis sobre la funcionalidad de los sitios web y su vinculación con la gobernanza educativa se asumió como un trasunto de gran complejidad multinivel (Spencer, 2017).

Un efecto de la evolución de dichos preceptos, lo mencionan Bejou \& Bejou (2016) sobre la aplicación de un sistema de gobernanza compartido genuino desarrollado por sus constituyentes que, en su praxis de más de 14 años, ha sido adoptada para consolidar un equilibrio y resultados sorprendentes sobre la excelencia académica, facilitando y apoyando el crecimiento de la universidad, así como expresión artística, académica e investigativa. Ahora bien, pasando al ámbito europeo, Giannone \& Santaniello (2018) mencionaron que el asunto es muy parecido al hecho anterior, ya que en su extensa revisión de estudios sobre los indicadores de aspectos políticos de la medición y el poder gubernamental de estados miembros de la Unión Europea (UE), en relación con las políticas digitales refleja una visión neoliberal del cómo debería ser la Europa digital. "It is hard to imagine any government function or governance process that does not involve extensive use of information and technology”. [Es difícil imaginar una función gubernamental o un proceso de gobernanza que no implique un uso extensivo de la información y la tecnología.] (Gil-García et al., 2017, p. 1).

De acuerdo al contexto de la dinámica de la gobernanza educativa de la región europea, han visto la enorme necesidad de la implementación, desarrollo y difusión de sitios web para poder gobernar el campo educativo, según lo investigado por Decuypere (2016). Un ejemplo viable para tener una idea sobre los aspectos de digitalización de la gobernanza educacional es lo que referenció Williamson (2016) acerca del portal denominado Education GPS (http:// gpseducation.oecd.org/), desarrollado por la OCDE que interactúa como portal de acceso público, por el cual se puede acceder a una vastedad de data a gran escala tales como encuestas, pruebas, entre otros. 
Sobre la gobernanza digital, política digital y educación

Williamson (2016) ha comentado que se han desarrollado una serie de trabajos relacionados con métodos científicos de datos que, a pesar de haber sido ensayados, reconocidos en importancia por la OCDE (2015), aún no hay una clara distinción entre aquello que funciona en relación con los enfoques de políticas científicas de datos que movilizan el conocimiento de las habilidades sociales y emocionales que puedan generar información conveniente para la política y práctica educativas. Quizás dicho asunto se aborda en su objetividad desde la óptica científica que visualiza aquello que funciona o es adecuado como el logro de una serie de configuraciones complejas, del empleo de estándares adecuados y un aparato de sistematización de la información con la consiguiente taxonomía de procesos; pero que en función del logro social aún dista de mantener un sesgo inclusivo como conocimiento relevante para las políticas, ya que la objetividad científica de los datos que busca la política educativa es el resultado de las prácticas de estandarización y cuantificación implementadas para resolver las controversias sobre la definición y medición de las cualidades humanas al convertirlas en categorías y números (Safavi \& Håkanson, 2016). En dicho sentido, aquello que funciona como entidad objetiva relevante para la política y conocimiento de la política científica de datos es el producto contingente de procesos prácticos, técnicos, que ejercen un poder e influencia considerables en política educativa (Williamson \& Piattoeva, 2018).

\section{Conclusiones}

Los enormes desafíos exigen tener consideraciones más extendidas acerca de la gobernanza de tipo multinivel para la gestión de una "ciudad inteligente". La ciudad inteligente se compone de atributos, temas e infraestructura (Silva et al., 2018), los mismos que se reconocen como características que son los pilares de una ciudad inteligente ya que proporciona plataformas digitales operativas que viabilizan su gobernanza y se fortalecen en infraestructuras institucionales, físicas y económicas. Este aspecto tan álgido requiere una óptica visionaria sobre la infraestructura digital que logre articular puntos de vista y perspectivas multiescala desde los diversos ángulos analíticos que se requieran como, por ejemplo, lo mencionado por De Gier (2018) desde la óptica internacional, los entes políticos han sido únicamente representantes de corporaciones tecnológicas con planteamientos de gestación de la urbanización planetaria, tal como es el caso del desarrollo y aplicación de las políticas de ancho de banda y la provisión de Internet en los principales núcleos urbanos, que viene a ser una tarea repartida entre las entidades políticas en función de sus representantes, las entidades comerciales (empresa, banca) y los usuarios como beneficiarios finales de tal coyuntura, actuando como consumidores a gran escala. El empleo de una serie de datos longitudinales permite comprender cómo las grandes empresas de datos cambian y configuran poderosamente formas híbridas de gobierno (Flyverbom et al., 2019). La noción de ciudad inteligente aún se encuentra en proceso de evolución ya que la experimentación e implementación de nuevos mecanismos se limitan dentro de los límites de los países desarrollados. (Fischer \& Malan, 2019; Rich, 2018; Silva et al., 2018). Por lo tanto, el desarrollo de los sistemas de gobernanza 
multinivel sustentada en infraestructuras digitales urbanas, es solo un primer escollo crítico a superar a fin de consolidar ecosistemas tecnológicos urbanos de mayor complejidad hacia infraestructuras digitales mucho más complejas y eficientes, por lo cual se propone que las denominadas infraestructuras digitales emergentes, sean parte de una prioritaria agenda de investigación centrada en las políticas de gobernanza urbana digital para las próximas décadas.

Los gobiernos enfrentan novedosas formas de adaptación y prestación de servicios a los ciudadanos ya que se pueden valer de las redes sociales para compartir información y movilizar o disolver el apoyo (White, 2019), además de tener acceso a plataformas colaborativas que facilitan su asistencia al público dentro y fuera del gobierno, independientemente del tiempo y la distancia. Al respecto, Pereira et al. (2019) manifestaron que el futuro de la evolución de Internet garantiza una nueva versión de los servicios de gobierno electrónico. Por su parte, los gobiernos pueden capitalizar nuevas fuentes de evidencia a fin de orientar los mecanismos de intervención política, para lo cual se puede valer de dos fuentes: la big data generada de las operaciones administrativas internas y actividades digitales de ciudadanos y gobiernos y, también de las propuestas de laboratorios de innovación, hackatones y avances en las ciencias sociales y la novedosa economía del comportamiento (Gibson, 2019; Clarke et al., 2018; Peng, 2017; Johnson, 2016).

Las investigaciones sobre las categorías clave de la denominada ciudadanía digital se orienta a satisfacer los requerimientos en los tiempos en que la identidad, las actividades rutinarias y el eje de gravedad de la vida política, económica, social y cultural está siendo construido en y alrededor de la internet. Se deben realizar estudios sociales orientados a sensibilizar acerca de la importancia de la ciudadanía digital como objetivo principal en la educación, ya que dichos estudios podrían respaldar un enfoque general de cómo podría ser la ciudadanía digital democrática y cómo sería la forma por la cual los maestros educarían a sus estudiantes para que se conviertan en ciudadanos digitales responsables, adecuadamente informados e involucrados activamente a nivel local, nacional y global (Flyverbom et al., 2019). En referencia a la ciudadanía deberán proponerse futuras investigaciones para desarrollar instrumentos capaces de medir habilidades, pensamientos y comportamientos de las personas en comunidades basadas en internet y también proponer elementos básicos de enseñanza de la ciudadanía digital en el desarrollo curricular.

La avanzada y rápida progresión de las tecnologías digitales ha contribuido a cambios estructurales masivos en la economía mundial; mas si se la enfoca desde la óptica de la gobernanza local, solo se ha generado una imagen sesgada de la problemática, tal como lo refieren Han y Jia (2018), ya que si la cantidad y calidad de la data procesada no es completa, en relación con las diversas agencias gubernamentales en una determinada localidad, puede afectar los mecanismos de gestión adecuados para dar una respuesta efectiva. Entre otras cosas, las investigaciones apuntan a las limitaciones que puedan generarse tanto de soporte (que 
Sobre la gobernanza digital, política digital y educación

pueden remediarse paulatinamente) y de vigilancia digital, que involucre a los ciudadanos y ejercer en cierta forma una represión de las expresiones en línea. La idea de emplear el poder de la tecnología orientada a potenciar un mecanismo de gobernanza pública sería una forma de democracia directa habilitada por la tecnología que permitiría a los ciudadanos limitar el accionar de los políticos y servidores públicos; pero ello aun no es viable ya que las evidencias sugieren, cada vez con mayor fuerza, que ello no funciona y se puede correr el riesgo de que los grupos de poder que puedan tener el control o explotación de las tecnologías de una forma más efectiva puedan imponer su voluntad a grupos minoritarios (Bannister, 2017).

\section{Referencias}

Aili, C. \& Nilsson, L. (2016). Preparing higher education students for the new landscape of governance. Tertiary Education and Management, 22(3), 249-265. doi: 10.1080/13583883.2016.1196725.

Akcil, U., Altinay, Z. \& Altinay, F. (2016). Assessing the Effects of Managers in the Digital Age on the Management Process of Digital Citizenship Roles. The Anthropologist, 23(1-2), 209217. doi: 10.1080/09720073.2016.11891943.

Alper, M. \& Goggin, G. (2017). Digital technology and rights in the lives of children with disabilities. New Media and Society, 19(5), 726-740. doi: 10.1177/1461444816686323.

Bannister, F. (2017). In Defence of Bureaucracy: Governance and Public Values in a Digital Age. Beyond Bureaucracy, 27-47. Doi: 10.1007/978-3-319-54142-6_3.

Barassi, V. (2019). Datafied Citizens in the Age of Coerced Digital Participation. Sociological Research Online. Doi: 10.1177/1360780419857734.

Barns, S. (2016). Mine your data: open data, digital strategies and entrepreneurial governance by code. Urban Geography, 37(4), 554-571. doi: 10.1080/02723638.2016.1139876.

Barns, S., Cosgrave, E., Acuto, M. \& Mcneill, D. (2016). Digital Infrastructures and Urban Governance. Urban Policy and Research, 35(1), 20-31. doi: 10.1080/08111146.2016.1235032.

Barreiro, B. (2018). How Can China Influence the Transatlantic Governance of Cultural Products in the Digital Age? The Journal of World Investment \& Trade, 19(3), 444-474. doi: 10.1163/22119000-12340096.

Bejou, D. \& Bejou, A. (2016). Shared Governance: The Key to Higher Education Equilibrium. Journal of Relationship Marketing, 15(1-2), 54-61. doi: 10.1080/15332667.2015.1091630.

Bellanova, R. (2016). Digital, politics, and algorithms. European Journal of Social Theory, 20(3), 329-347. doi: 10.1177/1368431016679167. 
Bianchi, I. S., \& Sousa, R. D. (2016). IT Governance Mechanisms in Higher Education. Procedia Computer Science, 100, 941-946. doi: 10.1016/j.procs.2016.09.253.

Botchway, E., Boateng, E. \& Kwofie, T. (2016). Benefits of e-governance implementation in physical infrastructure development at the local government level. Electronic Government, an International Journal, 12(4), 395-405. doi: 10.1504/eg.2016.080441.

Choi, M. (2016). A Concept Analysis of Digital Citizenship for Democratic Citizenship Education in the Internet Age. Theory \& Research in Social Education, 44(4), 565-607. doi: 10.1080/00933104.2016.1210549.

Clarke, A., Lindquist, E. \& Roy, J. (2017). Understanding governance in the digital era: An agenda for public administration research in Canada. Canadian Public Administration, 60(4), 457-475. doi: /10.1111/capa.12246.

Constantinides, P., Henfridsson, O. \& Parker, G. (2018). Introduction-Platforms and Infrastructures in the Digital Age. Information Systems Research, 29(2), 381-400. doi: 10.1287/isre.2018.0794.

Cushing, A. \& Shankar, K. (2018). Digital curation on a small Island: a study of professional education and training needs in Ireland. Archives and Records, 40(2), 1-18. Doi: 10.1080/23257962.2018.1425135.

da Silva, C., Ribeiro, Q., Soares, M. \& do Nascimento, R. (2019). ICT governance: A view of adoption of best practices in enterprises of sergipe state. ACM International Conference Proceeding Series. Doi: 10.1145/3330204.3330268.

Decuypere,M.(2016).DiagramsofEuropeanization:Europeaneducationgovernancein thedigital age. Journal of Education Policy, 31(6), 851-872. doi: 10.1080/02680939.2016.1212099.

De Gier, J. (2018). IT Governance of Dutch Municipalities and Digital Information Management. New Review of Information Networking, 23(1-2), 36-46. doi: 10.1080/13614576.2018.1537798.

Echalar, A. e Peixoto, J. (2017). Programa Um Computador por Aluno: o acesso às tecnologias digitais como estratégia para a redução das desigualdades sociais. Ensaio:Avaliação e Políticas Públicas em Educação, 25(95), 393-413. doi: 10.1590/s0104-40362017002501155.

Emejulu, A. \& McGregor, C. (2016). Towards a radical digital citizenship in digital education. Critical Studies in Education, 60(1), 1-17. doi: 10.1080/17508487.2016.1234494.

Evans, A. \& Gomes, R. (2017). The role of digital literacy in citizens' adoption of digital public services: The Portuguese case. Proceedings of the European Conference on e-Government, ECEG, 71-78.

Felix, R., Rauschnabel, P. \& Hinsch, C. (2017). Elements of strategic social media marketing: A holistic framework. Journal of Business Research, 70, 118-126. doi: 10.1016/j. jbusres.2016.05.001. 
Sobre la gobernanza digital, política digital y educación

Fernandez, S. (2019). Making space in higher education: disability, digital technology, and the inclusive prospect of digital collaborative making. International Journal of Inclusive Education, 1-16. doi: 10.1080/13603116.2019.1610806

Fischer, R. \& Malan, B. (2019). The Development of the Digital Wellness Toolkit. Association for Computing Machinery, 3(5), 329-337. doi: 10.1145/3326365.3326408.

Flyverbom, M., Deibert, R. \& Matten, D. (2019). The governance of digital technology, big data, and the internet: New roles and responsibilities for business. Business \& Society, 58(1), 3-19. doi: 10.1177/0007650317727540.

Fuente, C. (2017).Vulnerable publics and digital empowerment: The challenge of an e-inclusive society. Profesional de la Información, 26(1), 5-12. doi: 10.3145/epi.2017.ene.01.

Giannone, D. \& Santaniello, M. (2018). Governance by indicators: the case of the Digital Agenda for Europe. Information, Communication \& Society, 1-14. Doi: 10.1080/1369118x.2018.1469655.

Gibson, T. (2019). Digital humanities, libraries, and collaborative research: New technologies for digital textual studies. College \& Undergraduate Libraries, 1-29. Doi: 10.1080/10691316.2019.1638702.

Gil-Garcia, R., Dawes, S. \& Pardo, T. (2017). Digital government and public management research: finding the crossroads. Public Management Review, 20(5), 633-646. doi: 10.1080/14719037.2017.1327181.

Han, R. \& Jia, L. (2018). Governing by the Internet: local governance in the digital age. Journal of Chinese Governance, 3(1), 67-85. doi: 10.1080/23812346.2018.1429175.

Hansen, B. (2019). The digital revolution - digital entrepreneurship and transformation in Beijing. Small Enterprise Research, 26(1) 1-19. doi: 10.1080/13215906.2019.1570321.

Hazenberg, L. J. \& Zwitter, A. (2017). Network Governance im Big Data- und Cyber-Zeitalter. Zeitschrift Für Evangelische Ethik, 61(3), 184-209. doi: 10.14315/zee-2017-0305

Hepburn, P. (2018). A new governance model for delivering digital policy agendas: A case study of digital inclusion amongst elderly people in the UK. International Journal of E-Planning Research, 7(3), 36-49. doi: 10.4018/IJEPR.2018070103.

Huygh, T. \& De Haes, S. (2019). Investigating IT Governance through the Viable System Model. Information Systems Management, 36(2), 1-25. doi: 10.1080/10580530.2019.1589672.

Janowski, T., Estevez, E. \& Baguma, R. (2018). Platform governance for sustainable development: Reshaping citizen-administration relationships in the digital age. Government Information Quarterly. doi: 10.1016/j.giq.2018.09.002.

Johnson, B. (2016). Networked communication and the reprise of tolerance theory: civic 
education for extreme speech and private governance online. First Amendment Studies, 50(1), 14-31. doi: 10.1080/21689725.2016.1154478.

Ju, J., Liu, L. \& Feng,Y. (2019). Design of an O2O Citizen Participation Ecosystem for Sustainable Governance. Information Systems Frontiers. Doi: 10.1007/s10796-019-09910-4.

Knox, J., Williamson, B. \& Bayne, S. (2019). Machine behaviourism: future visions of "learnification" and "datafication" across humans and digital technologies. Learning, Media and Technology, (45)1, 1-15. Doi: 10.1080/17439884.2019.1623251.

Maltese, V., \& Giunchiglia, F. (2016). Foundations of Digital Universities. Cataloging \& Classification Quarterly, 55(1), 26-50. doi: 10.1080/01639374.2016.1245231.

Marland, A., Lewis, J. P. \& Flanagan, T. (2016). Governance in the Age of Digital Media and Branding. Governance, 30(1), 125-141. doi: 10.1111/gove.12194.

Neufeld, P. \& Delcore, H. (2018). Situatedness and variations in student adoption of technology practices: towards a critical techno-pedagogy. Journal of Information Technology Education: Research, 17, 1-38. doi: 10.28945/3934.

Nosthoff, A. \& Maschewski, F. (2019). The obsolescence of politics: Rereading Günther Anders's critique of cybernetic governance and integral power in the digital age. Thesis Eleven, 153(1), 75-93. doi: 10.1177/0725513619863853.

OECD. (2015). Skills for Social Progress: The Power of Social and Emotional Skills. Paris, France: OECD Skills Studies, OECD Publishing.

Ovando, M., Olivera, E. \& Bocarando, J. (2018). Before and after the reform: Fixed line internet penetration in Mexican households. Revista de Direito, Estado e Telecomunicações, 10(2), 1-14. doi: 10.26512/lstr.v10i2.21490.

Ozga, J. (2015). Trust in numbers? Digital Education Governance and the inspection process. European Educational Research Journal, 15(1), 69-81. doi: 10.1177/1474904115616629.

Paschoal, B. \& Wegrich, K. (2019). Urban governance innovations in Rio de Janeiro: The political management of digital innovations. Journal of Urban Affairs, 41(1), 117-134. doi: 10.1080/07352166.2017.1310561.

Peng,Y. (2017).Affective networks: howWeChat enhancesTencent's digital business governance. Chinese Journal of Communication, 10(3), 264-278. doi: 10.1080/17544750.2017.1306573

Pereira, G., Charalabidis, Y., Alexopoulos, C., Mureddu, F., Parycek, P., Ronzhyn, A...,. Wimmer, M. A. (2018). Scientific foundations training and entrepreneurship activities in the domain of ICT-enabled governance. Proceedings of the 19th Annual International Conference on Digital Government Research Governance in the Data Age - Dgo '18. Doi: $10.1145 / 3209281.3209316$. 
Sobre la gobernanza digital, política digital y educación

Pérez, L. \& Foronda, C. (2018). Digital governance and information technologies in local action groups (LAGs). Cogent Social Sciences, 4, 1528730. doi: 10.1080/23311886.2018.1528730.

Rich, E. (2018). Gender, health and physical activity in the digital age: between postfeminism and pedagogical possibilities. Sport, Education and Society, 23(8), 736-747. doi: 10.1080/13573322.2018.1497593.

Safavi, M. \& Håkanson, L. (2016). Advancing theory on knowledge governance in universities: a case study of a higher education merger. Studies in Higher Education, 43(3), 500-523. doi: 10.1080/03075079.2016.1180675.

Salemink, K., Strijker, D. \& Bosworth, G. (2017). Rural development in the digital age: a systematic literature review on unequal ICT availability, adoption, and use in rural areas. Journal of Rural Studies, 54, 360-371. doi: 10.1016/j.jrurstud.2015.09.001.

Schou, J. \& Hjelholt, M. (2018a). Digital state spaces: state rescaling and advanced digitalization. Territory, Politics, Governance, 7(4) 1-17. Doi: 10.1080/21622671.2018.1532809.

Schou, J. \& Hjelholt, M. (2018b). Digital citizenship and neoliberalization: governing digital citizens in Denmark. Citizenship Studies, 22(5), 507-522. doi: 10.1080/13621025.2018.1477920.

Silva, B. N., Khan, M., \& Han, K. (2018). Towards sustainable smart cities: A review of trends, architectures, components, and open challenges in smart cities. Sustainable Cities and Society, 38, 697-713. doi: 10.1016/j.scs.2018.01.053.

Singh, R. (2019). Give Me a Database and I Will Raise the Nation-State. South Asia. Journal of South Asian Studies, 42(3), 501-518. doi: 10.1080/00856401.2019.1602810.

Sixin, X., Yayuan, Y., Jiang, Y. \& Yue, W. (2017). A New Governance Architecture for Government Information Resources Based on Big Data Ecological Environment in China. 2017 IEEE International Symposium on Multimedia (ISM). Doi: 10.1109/ism.2017.103.

Spante, M., Hashemi, S., Lundin, M. \& Algers, A. (2018). Digital competence and digital literacy in higher education research: Systematic review of concept use. Cogent Education, 5(1), 1-21. doi: 10.1080/2331186X.2018.1519143.

Spencer, S. (2017). Multi-level governance of an intractable policy problem: migrants with irregular status in Europe. Journal of Ethnic and Migration Studies, 44(12), 2034-2052. doi: $10.1080 / 1369183 \times .2017 .1341708$.

Touchton, M., Wampler, B., \& Spada, P. (2019). The digital revolution and governance in Brazil: Evidence from participatory budgeting. Journal of Information Technology \& Politics, 16(2), 154-168. doi: 10.1080/19331681.2019.1613281.

White, P. (2019). Cyberpeace: Why Internet Governance Matters for Global Peace and Stability. Peace \& Change. Doi: 10.1111/pech.12373. 
Williamson, B. (2015). Digital education governance: an introduction. European Educational Research Journal, 15(1), 3-13. doi: 10.1177/1474904115616630.

Williamson, B. (2016). Digital education governance: data visualization, predictive analytics, and "real-time" policy instruments. Journal of Education Policy, 31(2), 123-141. doi: 10.1080/02680939.2015.1035758.

Williamson, B. \& Piattoeva, N. (2018). Objectivity as standardization in data-scientific education policy, technology and governance. Learning, Media and Technology, 1-13. doi: 10.1080/17439884.2018.1556215.

Zalnieriute, M. \& Milan, S. (2019). Internet Architecture and Human Rights: Beyond the Human Rights Gap. Policy \&Internet, 11(1), 6-15. doi: 10.1002/poi3.200.

Zhao, W. (2017). “Observation” as China's civic education pedagogy and governance: an historical perspective and a dialogue with Michel Foucault. Discourse: Studies in the Cultural Politics of Education, 40(6), 1-14. doi: 10.1080/01596306.2017.1404444.

Zhu, H. \& Peters, M. A. (2018). Social governance, education and socialist rule of law in China. Educational Philosophy and Theory, 51(7), 1-4. doi: 10.1080/00131857.2018.1482643. 